\title{
Primary care-based intervention to reduce at-risk drinking in older adults: a randomized controlled trial
}

\author{
Alison A. Moore', Fred C. Blow ${ }^{2}$, Marc Hoffing ${ }^{3}$, Sandra Welgreen ${ }^{4}$, James W. Davis ${ }^{5}$, \\ James C. Lin ${ }^{6}$, Karina D. Ramirez ${ }^{5}$, Diana H. Liao ${ }^{5}$, Lingqi Tang ${ }^{7}$, Robert Gould ${ }^{8}$, Monica Gill' \\ Oriana Chen ${ }^{10}$ \& Kristen L. Barry ${ }^{2}$
}

Department of Medicine, Department of Psychiatry and Biobehavioral Sciences, University of California at Los Angeles, Los Angeles, CA, USA,' Department of Psychiatry, University of Michigan and Veterans Affairs National Serious Mental Illness Treatment Research and Evaluation Center (SMITREC), Ann Arbor, MI, USA, ${ }^{2}$ Desert Oasis Healthcare, Palm Springs, CA, USA, ${ }^{3}$ Kaiser Permanente, Southern California, Panorama City, CA, USA, ${ }^{4}$ Department of Medicine, University of California at Los Angeles, Los Angeles, CA, USA, ${ }^{5}$ Veterans Affairs Greater Los Angeles Healthcare Systems, Los Angeles, California, Department of Medicine, Cheng Ching Hospital, Taichung, Taiwan, ${ }^{6}$ Department of Psychiatry and Biobehavioral Sciences, Health Services Research Center, University of California at Los Angeles, Los Angeles, CA, USA, ${ }^{7}$ Department of Statistics, University of California at Los Angeles, Los Angeles, CA, USA, ${ }^{8}$ Eastern Virginia Medical School, Norfolk, VA, USA ${ }^{9}$ and College of Medicine, Northeastern Ohio Universities Colleges of Medicine and Pharmacy (NEOUCOM), Rootstown, OH, USA ${ }^{10}$

\begin{abstract}
Aims To examine whether a multi-faceted intervention among older at-risk drinking primary care patients reduced at-risk drinking and alcohol consumption at 3 and 12 months. Design Randomized controlled trial. Setting Three primary care sites in southern California. Participants Six hundred and thirty-one adults aged $\geq 55$ years who were at-risk drinkers identified by the Comorbidity Alcohol Risk Evaluation Tool (CARET) were assigned randomly between October 2004 and April 2007 during an office visit to receive a booklet on healthy behaviors or an intervention including a personalized report, booklet on alcohol and aging, drinking diary, advice from the primary care provider and telephone counseling from a health educator at 2, 4 and 8 weeks. Measurements The primary outcome was the proportion of participants meeting at-risk criteria, and secondary outcomes were number of drinks in past 7 days, heavy drinking (four or more drinks in a day) in the past 7 days and risk score. Findings At 3 months, relative to controls, fewer intervention group participants were at-risk drinkers [odds ratio (OR) 0.41; $95 \%$ confidence interval (CI) 0.22-0.75]; they reported drinking fewer drinks in the past 7 days [rate ratio (RR) $0.79 ; 95 \%$ CI 0.700.90], less heavy drinking (OR 0.46; 95\% CI 0.22-0.99) and had lower risk scores (RR 0.77 95\% CI 0.63-0.94). At 12 months, only the difference in number of drinks remained statistically significant (RR 0.87; 95\% CI 0.76-0.99). Conclusions A multi-faceted intervention among older at-risk drinkers in primary care does not reduce the proportions of at-risk or heavy drinkers, but does reduce amount of drinking at 12 months.
\end{abstract}

Keywords Aged, alcohol, comorbidity, intervention, primary care, screening.

Correspondence to: Alison A. Moore, Division of Geriatric Medicine, David Geffen School of Medicine at UCLA, 10945 Le Conte Boulevard, Suite 2339, Los Angeles, CA 90095, USA. E-mail: aamoore@mednet.ucla.edu

Submitted 23 June 2010; initial review completed 27 August 2010; final version accepted 17 September 2010

\section{INTRODUCTION}

At-risk drinking falls within the spectrum of unhealthy alcohol use [1] and is defined typically as exceeding particular drinking limits [2-4]. One definition of at-risk drinking is consuming $>14$ standard drinks per week or more than four drinks per occasion for men less than 65 years of age and more than seven drinks per week or more than three drinks per occasion for women and people aged 65 years and older [5]. Using this definition, between 2 and $3 \%$ of women and $9-10 \%$ of men aged 65 years and older engage in at-risk drinking $[2,6,7]$.

Older adults have risks associated with drinking that differ somewhat from younger people because of age-related physiological changes that increase blood alcohol levels for a given dose, increased brain sensitivity to alcohol and increases in morbidity and medication use [8-10]. A definition of at-risk drinking more relevant to 
older adults is the use of alcohol that increases risk for harm, due both to the amount consumed and concurrent use of alcohol with medications and comorbidities [11].

Using a definition of at-risk drinking that includes alcohol use and comorbidity, $18 \%$ of men and $5 \%$ of women aged 60 years and older were at-risk drinkers in a population-based sample of US adults [12]. At-risk male drinkers had a $20 \%$ higher mortality rate than not at-risk drinkers.

Randomized controlled trials of brief alcohol interventions have been proven to reduce unhealthy alcohol use in primary care populations[13], but few have focused on older adults [14,15]. No randomized controlled trial has tested interventions to reduce risks associated with alcohol, comorbidity and medication use in older adults.

The Comorbidity Alcohol Risk Evaluation Tool (CARET), whose precursor is the short Alcohol-Related Problems Survey [16], is a measure to identify at-risk drinking older adults. The CARET takes 2-5 minutes to self-administer and uses information on amount of alcohol use, comorbidity, symptoms and medications to assess drinking risks [16-20]. One study used a related instrument to identify older adults as harmful, hazardous or non-hazardous drinkers in three sites within a primary care practice [15]. At site 1 , reports outlining patients' drinking risks were sent to both physicians and patients; at site 2, reports were sent only to patients; and at site 3, no reports were sent. Patients receiving reports had reduced drinking risks and those at site 1 also reduced drinking amount compared to site 3 at 12 months.

While these data were promising, we conducted a more rigorous trial by randomizing older at-risk drinkers to intervention or control groups, including multiple primary care practices in three separate organizations, and utilizing an intervention containing components proven effective to change behavior [13,14,21]. We hypothesized that, compared to the control group, the intervention would cause greater reductions in both at-risk drinking and alcohol consumption among older adults in primary care settings.

\section{METHODS}

\section{Study design}

The Healthy Living As You Age (HLAYA) study is a randomized clinical trial testing the effectiveness of a screening and multi-component intervention among older at-risk drinkers in community-based primary care settings. The primary aims of the study were to reduce at-risk drinking and alcohol use at 12 months. The Institutional Review Boards from the University of California at Los Angeles and Kaiser Permanente Southern California approved this study. All enrolled participants provided written informed consent.

\section{Setting and primary care providers (PCP)}

Study sites included three organizations in Southern California: an independent provider organization, Desert Oasis Healthcare (DOHC); a large group model health maintenance organization: Kaiser Permanente Southern California (KPSC); and a university-affiliated ambulatory care system, UCLA Healthcare. Primary care providers (PCP) practising at the study sites were recruited in 2004 through announcements and e-mails sent by study authors (M.H., S.W., A.M.). The 145 participating PCPs ( $48.6 \%$ of those eligible) received 30 minutes' instruction by an investigator (A.M.) in the study procedures and how to provide brief advice (1-5 minutes) to reduce drinking modeled after the National Institute on Alcohol Abuse and Alcoholism publication Helping Patients Who Drink Too Much: A Clinician's Guide [5].

\section{Participants}

Participants were recruited from October 2004 to April 2007 and identified from a list of patients scheduled to see a participating PCP in the following week. To be eligible for screening, participants had to be aged 55 years or older, speak English or Spanish, see participating clinicians and report having consumed at least one alcoholic drink in the past week. Those who could not hear the screening questions, were too ill to participate or had received treatment for an alcohol use disorder in the prior 3 months were ineligible. Clinical staff and volunteers telephoned 7478 patients who met the age eligibility criterion to introduce the study as one focusing on healthy behaviors in older adults and to screen for eligibility. Of those contacted, 2138 (29\%) were eligible to be screened further for study enrollment (Fig. 1).

\section{Identification of at-risk drinkers}

Those agreeing to participate in the secondary screening were administered the CARET over the telephone. The CARET includes questions assessing past 12-month (i) quantity and frequency of drinking, (ii) episodic heavy drinking (four or more drinks on an occasion), (iii) driving within 2 hours of drinking three or more drinks, (iv) others being concerned about the respondent's drinking, (v) medical and psychiatric conditions, (vi) symptoms that could be caused or worsened by alcohol and (vii) medications that could interact negatively with or whose efficacy could be diminished by alcohol.

Responses were scored to designate a participant as either an at-risk (score 1-7) or not at-risk drinker (score 


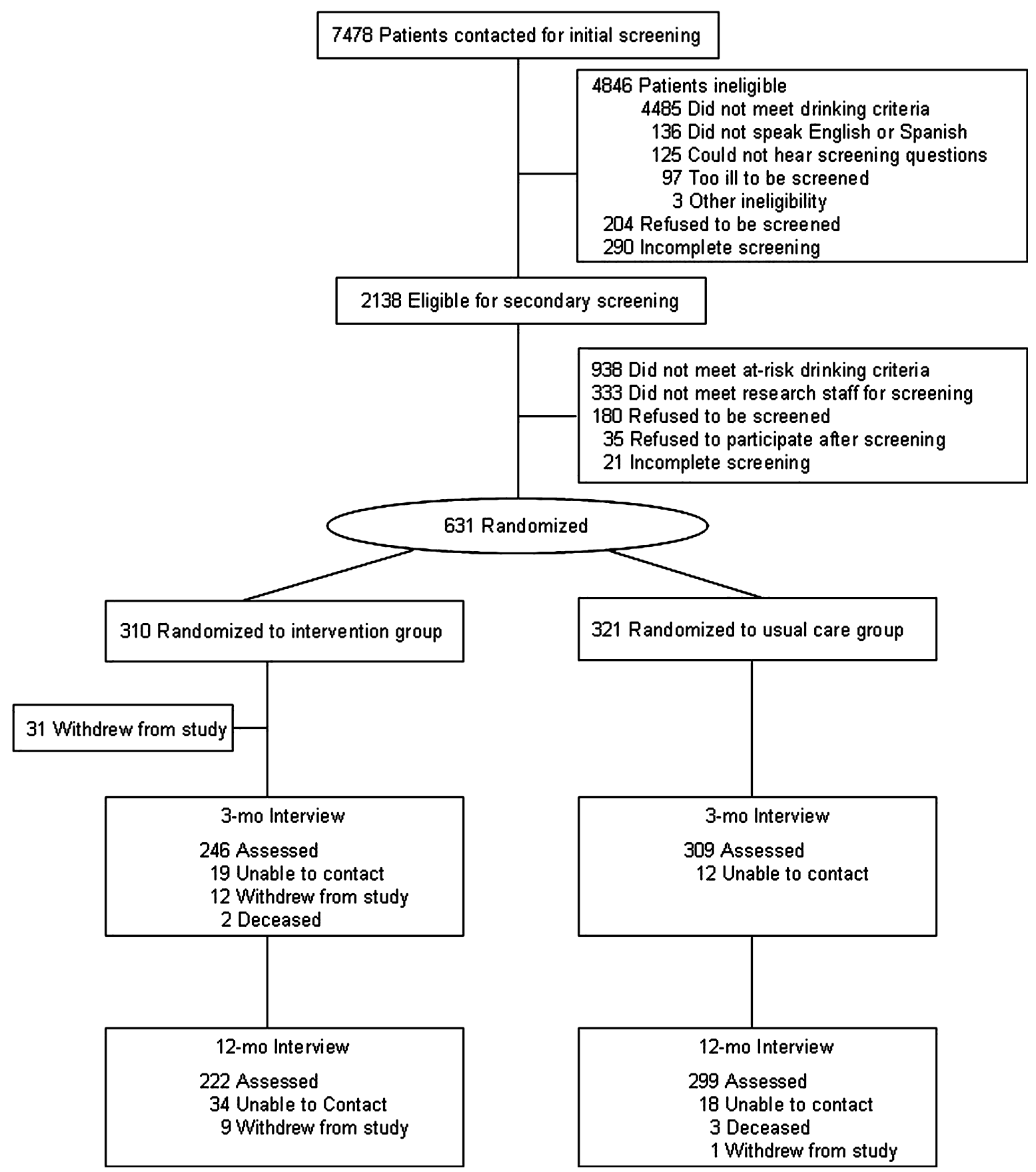

Figure I Flow of individuals in the Healthy Living As You Age (HLAYA) trial

$0)$. Some examples of at-risk drinkers include people who report (i) drinking three drinks four or more times a week, or (ii) drinking two or more drinks at least twice a week and often having heartburn, or (iii) drinking two drinks daily and taking alprazolam at least three to four times a week. Of the 2138 individuals eligible for secondary screening, 631 (30\%) were at-risk drinkers and agreed to participate in the trial. Approximately 1 week later, at the time of a regular visit with their PCPs (i.e. baseline visit), these participants were assigned randomly to the intervention group (310 individuals) or control group (321 individuals).

Randomization and blinding

Research assistants used consecutively numbered, sealed opaque envelopes containing assignment information prepared at the study coordinating center using a computer-generated set of random numbers. Randomization of participants was stratified by site and gender; 
within each stratum randomly sized blocks of 8,10 or 12 were used. Within each block, equal numbers were assigned to intervention or control groups. PCPs could see patients in both groups, but they were alerted only when seeing intervention group subjects. Research assistants, blinded to treatment allocation, conducted all baseline and follow-up outcome assessments. To legitimize alcohol use as a health issue [22], and to mask the true purpose of the study to potential participants before randomization occurred, questions on seat belt use, exercise, diet and smoking were asked.

\section{Control group}

At the baseline visit control group participants received a booklet outlining recommended behaviors for alcohol use, nutrition, exercise, medication use and smoking. Research assistants encouraged participants to read the booklet and discuss it with their PCPs.

\section{Intervention group}

At the baseline visit the research assistant informed intervention group participants that the study focused on healthy drinking behaviors and they were asked to sign a second informed consent form describing additional procedures. Before seeing the PCP, the research assistant handed each participant a personalized report and drinking diary to keep track of alcohol use. A similar version of the report was given to the PCP. The reports outlined participants' alcohol-associated risks identified by the CARET (e.g. drinking two drinks daily and taking a sedative) and potential consequences (e.g. increased sedation and falls). The PCP gave the participant oral and written advice (in prescription-style format on an alcohol education booklet). At 2, 4 and 8 weeks after the baseline visit, a health educator called participants to provide additional feedback and counseling using motivational interviewing techniques [23] to help reduce alcohol consumption. We have reported previously on this component of the intervention [24].

\section{Outcomes and follow-up}

Baseline questionnaires assessing demographic, healthrelated and alcohol consumption characteristics were completed before randomization. Health-related characteristics included: self-rated health status (excellent, very good, good, fair and poor), ability to perform strenuous activities and heavy work [25] and overall quality of life (0, as bad as dying, to 10 , perfect health). Alcohol consumption was assessed using the CARET and the 7-day alcohol time-line follow-back (TLFB) [26].

Follow-up assessments were made by telephone at 3 and 12 months after baseline. The CARET was adminis- tered using the time-frame since the prior assessment (e.g. at 3 months, questions were framed using 'During the past 3 months' as the question stem). The 7-day alcohol TLFB, questions on self-rated health status, ability to perform strenuous activities and heavy work and quality of life were re-administered. Participant follow-up concluded in July 2008. The amount of time spent conducting each assessment was 15-20 minutes for baseline, 10-15 minutes for the 3 month and 15-20 minutes for the 12-month assessment.

\section{Statistical analysis}

As there were no available data on changes in CARETdefined at-risk drinking, we based the sample size estimates on two trials of brief advice in primary care $[14,27]$ using number of drinks per week as the outcome. We anticipated that our enrolled sample would be drinking less than in other trials, as participants could be identified as at-risk drinkers while drinking one to two drinks per day depending on their comorbidities and medications used. The power calculations were based on expecting an average difference of 1.8-2.0 drinks per week between groups at 12 months and a standard deviation of 9.0-10.0. With 250 individuals per group, $80 \%$ power was projected to detect the effect size of 0.2 with alpha level of 0.05 (two-sided). Enrolling 313 individuals per group allowed up to $20 \%$ attrition rate.

Analyses of outcome data were based on the intention-to-treat method and measured at the 3- and 12-month follow-up periods. The outcomes were the: (1) percentage of participants meeting at-risk criteria on the CARET (yes or no); (2) number of risks identified on the CARET (range 0-7); (3) number of drinks consumed per week as measured by the 7-day alcohol TLFB; and (4) heavy drinking (four or more drinks in a day) in the past 7 days (yes or no) using the TLFB,

Baseline characteristics were reported for the total sample and by group. Categorical data were reported as frequencies (percentages) and count data were reported as means and standard deviations (SD).

Among the 631 participants, 555 (88\%) completed the 3-month follow-up and 521 (83\%) completed the 12-month follow-up (Fig. 1). Of those in the intervention arm, $249(80.3 \%)$ completed at least one call with the health educator [24]. At 3 months, 246 (79\%) of the intervention group and 309 (96\%) of the control group completed the assessments; at 12 months, 222 (72\%) of the intervention group and 299 (93\%) of the control group completed the assessments $(P<0.05$ for both follow-ups).

Using data from the 3- and 12-month follow-ups, we conducted longitudinal data analyses using the Stata version $11.0 \mathrm{xt}$ series of commands (StataCorp, College 
Station, TX, USA). The number of drinks consumed in the past 7 days was analyzed with a negative binomial model using the xtenbreg procedure; the number of risks was analyzed by Poisson models with the xtpoisson procedure [28]. Logistic regression models were fitted for the dichotomous outcomes: heavy drinking in past 7 days and at-risk drinking status. All models included a random intercept to account for clustering within participant [29] and examined the fixed effects for group, follow-up assessment and their interaction with regression adjustment for age, gender, baseline measure for the same outcome and study site. The results of the negative binomial regression and Poisson model analyses are presented as rate ratios $(\mathrm{RR})$ and the logistic regression models as odds ratios (OR).

Because of differences in the magnitude of missing data between treatment groups, multiple imputation $[30,31]$ was used to address missing data for participants who did not complete the 3- or 12-month follow-up assessments using a multivariate normal model separately for each group. The model included outcome variables assessed at baseline, 3 and 12 months, and baseline covariates using the MI procedure in SAS version 9.2 (SAS Institute, Cary, NC, USA). We reported results from unimputed data along with multiple imputation as sensitivity analyses.

\section{RESULTS}

\section{Baseline sample characteristics}

In the sample, $63 \%$ were recruited from DOHC $(n=399)$, $21 \%$ from UCLA $(n=133)$ and $16 \%$ from KPSC $(n=99)$. They ranged in age from 55 to 89 years (Table 1 ). Most were men, non-Hispanic white, highly educated, retired, married and living with someone. Most reported their health as good or better, being able to perform both strenuous activities and heavy work, and good quality of life.

On average, in the past 7 days, participants reported drinking $>14$ drinks and more than a third drank more than four drinks in a day (Table 1). Participants had an average of three risks; $28 \%$ had just one risk, and $19 \%$, $14 \%, 18 \%$ and $21 \%$ had two, three, four or five or more risks, respectively. Most were identified as at-risk drinkers because of alcohol use with medications (73\%), followed by alcohol use with particular symptoms (60\%), alcohol use with comorbidities (50\%), amount of alcohol use alone (47\%), binge drinking (24\%), driving after drinking (24\%) and others being concerned about participant's drinking (17\%).

Among those identified as at-risk due to amount of alcohol consumption with comorbidity, most had hypertension, followed by depression, gout and diabetes
(Table 2). Among those identified as at-risk due to amount of alcohol consumption with particular symptoms, most were identified due to problems sleeping, gastrointestinal symptoms or memory problems. Among those identified as at-risk due to amount of alcohol consumption with medications, most were taking antihypertensives, followed by ulcer medications, non-steroidal anti-inflammatory drugs and antiplatelet drugs.

\section{Outcomes}

The percentage of at-risk drinkers declined steeply from baseline to 3 months in both groups, and was generally sustained at 12 months (Fig. 2). Amount of drinking and prevalence of heavy drinking days dropped precipitously from baseline to 3 months for both groups and these reductions were generally sustained at 12 months (Figs 3 and 4). The summary outcome data at 3 and 12 months are presented in Table 3. The between group difference of 1.3 drinks in the past 7 days observed at 12 months is smaller than the 1.8-2.0 drink difference that we expected when designing the study, and may not be clinically meaningful.

Table 4 presents treatment effects on the outcomes at 3 and 12 months, with and without imputation for missing values. At 3 months, relative to controls, fewer participants receiving the intervention were at-risk drinkers (OR 0.41; 95\% CI 0.22-0.75); they had lower risk scores (RR $0.7795 \%$ CI 0.63-0.94), reported drinking fewer drinks (RR 0.79; 95\% CI 0.70-0.90) and less heavy drinking (OR 0.46 ; 95\% CI $0.22-0.99$ ). At 12 months, the group differences in number of drinks in the past 7 days remained (RR $0.87 ; 95 \%$ CI $0.76-0.99$ ). Multiple imputation produced similar results as those without imputation (Table 4).

\section{DISCUSSION}

This randomized trial demonstrated that, compared to receiving an educational booklet, a multi-component intervention among older adults in primary care did not reduce at-risk drinking but did reduce alcohol consumption. In both intervention and control groups, prevalence of at-risk drinking declined by 50-60\%, amount of drinking declined by 30-40\% and prevalence of heavy drinking days declined by $30-70 \%$ from baseline to 3 months, and largely persisted at 12 months. In addition, those in the intervention group receiving personalized feedback about alcohol-associated risks had greater reductions in at-risk drinking, number of risks, amount of drinking and prevalence of heavy drinking days compared to the control group at 3 months; these differences continued to favor the intervention group but remained statistically significant only for amount of drinking at 12 months. 
Table 1 Baseline patient characteristics. ${ }^{\mathrm{a}}$

\begin{tabular}{|c|c|c|c|}
\hline Characteristic & Total $(n=631)$ & Intervention $(n=310)$ & Control $(n=321)$ \\
\hline Age, mean (SD) & $68.4(6.9)$ & $68.7(6.8)$ & $68.1(6.9)$ \\
\hline Male gender & $448(71)$ & $222(72)$ & $226(70)$ \\
\hline \multicolumn{4}{|l|}{ Race } \\
\hline White, non-Hispanic & $551(87)$ & $272(88)$ & $279(87)$ \\
\hline Hispanic/Latino & $58(9)$ & $27(9)$ & $31(10)$ \\
\hline Other & $21(3)$ & $10(3)$ & $11(3)$ \\
\hline \multicolumn{4}{|l|}{ Education } \\
\hline High school or less & $144(23)$ & $72(23)$ & $72(23)$ \\
\hline Some college & $194(31)$ & $99(32)$ & $95(30)$ \\
\hline College degree or more & $289(46)$ & $138(45)$ & $151(47)$ \\
\hline \multicolumn{4}{|l|}{ Marital status } \\
\hline Married & $311(50)$ & $153(50)$ & $158(50)$ \\
\hline Widowed, divorced, separated & $170(27)$ & $78(25)$ & $92(29)$ \\
\hline Never married & $145(23)$ & $76(25)$ & $69(22)$ \\
\hline \multicolumn{4}{|l|}{ Living arrangement } \\
\hline Living with spouse, partner or others & $464(74)$ & $228(74)$ & $236(74)$ \\
\hline Living alone & $165(26)$ & $81(26)$ & $84(26)$ \\
\hline \multicolumn{4}{|l|}{ Employment status } \\
\hline Retired or homemaker & $463(74)$ & $228(74)$ & $253(73)$ \\
\hline Working full- or part-time & $166(26)$ & $80(26)$ & $86(27)$ \\
\hline \multicolumn{4}{|l|}{ Self-rated health status } \\
\hline Excellent or very good & $328(52)$ & $156(50)$ & $172(54)$ \\
\hline Good & $233(37)$ & $123(40)$ & $110(34)$ \\
\hline Fair or poor & $69(11)$ & $31(10)$ & $38(12)$ \\
\hline \multicolumn{4}{|l|}{ Able to do } \\
\hline Strenuous activities & $471(75)$ & $226(73)$ & $245(77)$ \\
\hline Heavy work & $525(83)$ & $256(83)$ & $269(84)$ \\
\hline Self-rated quality of life (range -10), mean (SD) & $7.9(1.6)$ & $7.9(1.6)$ & $7.9(1.7)$ \\
\hline Number of drinks in past 7 days, mean (SD) & $15.2(7.3)$ & $15.1(7.2)$ & $15.2(7.4)$ \\
\hline Had a heavy drinking day in past 7 days ${ }^{\mathrm{b}}$ & $216(34)$ & $106(34)$ & $110(34)$ \\
\hline Risk score (range 0-7), mean (SD) & $2.9(1.7)$ & $2.9(1.7)$ & $3.0(1.7)$ \\
\hline \multicolumn{4}{|l|}{ Type of risk } \\
\hline Quantity and frequency of drinking ${ }^{\mathrm{c}}$ & $295(47)$ & $147(47)$ & $148(46)$ \\
\hline Binge (four or more drinks per occasion) drinking & $149(24)$ & $79(25)$ & $70(22)$ \\
\hline Driving after drinking & $154(24)$ & $73(24)$ & $81(25)$ \\
\hline Others being concerned & $106(17)$ & $50(16)$ & $56(17)$ \\
\hline Alcohol use with medical, psychiatric condition & $315(50)$ & $154(50)$ & $161(50)$ \\
\hline Alcohol use with symptoms & $378(60)$ & $185(60)$ & $193(60)$ \\
\hline Alcohol use with medications & $462(73)$ & $222(72)$ & $240(75)$ \\
\hline
\end{tabular}

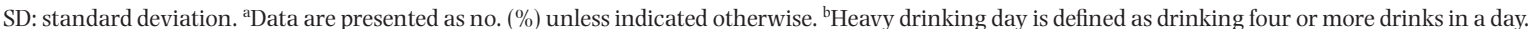
'Those meeting criterion for this risk drink three or more drinks per occasion at least four times a week, four or more drinks per occasion at least twice monthly or five or more drinks per occasion at any frequency.

In Project GOAL [14], the number of drinks consumed in the past 7 days for both study arms at baseline was similar to our sample, but alcohol consumption did not decline from baseline in the control group. This finding contrasts with our study and most others employing brief interventions $[13,32]$. Project GOAL's intervention was more intensive than ours, as physicians used a work-book to give advice to patients and the study included a second reinforcement visit with the physician, and included two follow-up calls from a clinic nurse 2 weeks after each physician visit. These features probably contributed to its success in reducing both 7-day alcohol use and binge drinking in the intervention group at 3, 6 and 12 months.

In the study by Fink et al. [15], respondents were classified as harmful, hazardous or non-hazardous drinkers using a scoring method similar to the CARET. At 12 months both groups receiving reports had overall lowerrisk classifications compared to the group not receiving a report. Our study did not have statistically significant differences in the proportions of those at-risk in the two study arms at 12 months possibly because those in the control arm received information on healthy drinking 
(similar to Project GOAL), and therefore received minimal intervention, while the study by Fink et al. gave no information on healthy drinking to the no report group.

The trial's data illustrate the unique risks of this sample of older adults who drink alcohol. They frequently used medications whose efficacy may be diminished by alcohol or may interact negatively with alcohol, such as antihypertensives, ulcer medications, non-steroidal antiinflammatory drugs and antiplatelet drugs. They also frequently had conditions or symptoms that may be

Table 2 Baseline comorbidities, symptoms and medications that increase risk with alcohol consumption.

\begin{tabular}{lr}
\hline Type of risk & $n(\%)$ \\
\hline Alcohol consumption with comorbidity & \\
Hypertension & $193(30.6)$ \\
Depression & $78(12.4)$ \\
Gout & $48(7.6)$ \\
Diabetes & $33(5.2)$ \\
Ulcer disease & $26(4.1)$ \\
Liver condition & $22(3.5)$ \\
Pancreatitis & $4(0.6)$ \\
Alcohol consumption with symptoms & \\
Problems sleeping & $243(38.5)$ \\
Gastrointestinal symptoms & $152(24.1)$ \\
Memory problems & $142(22.5)$ \\
Feeling sad or blue & $106(16.8)$ \\
Tripping, falling or having accidents & $112(17.8)$ \\
Alcohol consumption with medications & \\
Antihypertensives & $200(31.7)$ \\
Ulcer medications & $115(18.2)$ \\
Non-steroidal anti-inflammatory drugs & $113(17.9)$ \\
Antiplatelet drugs & $109(17.3)$ \\
Non-prescription medications for allergies & $80(12.7)$ \\
$\quad$ or sleep & \\
Antidepressants & $75(11.9)$ \\
Sedatives & $64(10.1)$ \\
Opioids & $42(6.7)$ \\
Nitrates & $27(4.3)$ \\
Warfarin & $28(4.4)$ \\
Seizure medications & $4(0.6)$ \\
\hline & \\
\hline
\end{tabular}

caused or worsened by alcohol such as hypertension, depression, insomnia, memory problems and gastrointestinal symptoms such as heartburn.

Regression towards the mean, social desirability bias and assessment effects may have influenced our study's findings. Because the intervention group was told the study focused on drinking it is possible that, relative to the blinded control group, they may have under-reported their alcohol consumption on follow-up assessments. We aimed to minimize this bias by designing other aspects of the study to reduce such bias (e.g. confidential reporting, non-judgmental nature of assessments). Both study groups completed three separate assessments and this may have reduced the effect of the intervention relative to the control condition [33,34].

One of the study's limitations, common among studies of brief alcohol interventions, was the differential attrition rate between the two study arms [13]. Although we aimed to reduce dropout in the intervention arm by offering those participants who seemed unwilling to receive health educator calls the option of no further health educator calls, there was a $17 \%$ and $21 \%$ difference in follow-up rates between the two study arms at 3 and 12 months, respectively. It could be that older adults who are identified as at-risk drinkers are wary of talking about a sensitive topic with someone they do not know. Counseling delivered by health professionals known to the older adult, as in Project GOAL, may be more successful in changing behavior. Also, the sample was primarily white, male, highly educated and in good health, and the study findings may not be generalizable to other populations. However, the sample is typical of older adults in community-based primary care who participate in brief alcohol interventions [14,15], and the general population of older adults who drink alcohol $[11,35,36]$. Some clinical staff conducted pre-screening and administered the baseline assessments for the project. Although some practices use clinical staff for such health behavior assessments, it is not standard of care and could be challenging for many primary care practices to implement. Another
Figure 2 Percentage of at-risk drinkers. Error bars indicate 95\% confidence intervals







Figure 3 Mean number of drinks in the past 7 days. Error bars indicate standard deviations

Figure 4 Prevalence of heavy drinking episodes in the past 7 days. Error bars indicate $95 \%$ confidence intervals

Table 3 Summary outcome data at 3 and 12 months.

\begin{tabular}{|c|c|c|}
\hline & Intervention group & Control group \\
\hline Variable & Percentage & \\
\hline \multicolumn{3}{|l|}{ At-risk drinker, $\%$} \\
\hline 3 months (control group, $n=309$, intervention group, $n=246$ ) & 49.6 & 61.2 \\
\hline 12 months (control group, $n=299$, intervention group, $n=222$ ) & 54.1 & 59.9 \\
\hline \multicolumn{3}{|l|}{ One or more heavy drinking days in past 7 days } \\
\hline 3 months (control group, $n=296$, intervention group, $n=234$ ) & 10.3 & 16.9 \\
\hline \multirow[t]{2}{*}{12 months (control group, $n=294$, intervention group, $n=213$ ) } & 10.8 & 13.3 \\
\hline & Mean (SD) & \\
\hline \multicolumn{3}{|l|}{ Risk score (range $0-7$ ) } \\
\hline 3 months & $1.30(1.8)$ & $1.66(1.9)$ \\
\hline 12 months & $1.38(1.8)$ & $1.63(1.9)$ \\
\hline \multicolumn{3}{|l|}{ Number of drinks in past 7 days } \\
\hline 3 months & $8.93(7.3)$ & $10.73(8.0)$ \\
\hline 12 months & $9.39(8.0)$ & $10.70(8.4)$ \\
\hline
\end{tabular}

SD: standard deviation.

potential limitation is that PCPs could see participants in both the treatment and control arms of the study and could 'contaminate' the control arm by providing advice to reduce drinking. Similar to the control arm in Project GOAL, at the 3-month follow up 47 (15\%) control participants reported discussing alcohol use with their PCPs. Also, as all study participants received information on low-risk drinking limits, all received some type of intervention. Both these design characteristics may have reduced the observed differences between intervention and control groups. Finally, because we powered our study to detect differences in amount of drinking, it may have been underpowered to detect a difference in the prevalence of at-risk drinking. We did not assess whether participants met DSM criteria for alcohol abuse and/or dependence, but the relatively brief intervention conducted in this study may be unlikely to address the needs of those who have such alcohol use disorders. 
Table 4 Intervention effects on at-risk drinking and amount of alcohol consumption based on random-effects models with and without imputation for missing values.

\begin{tabular}{|c|c|c|}
\hline \multirow[b]{3}{*}{ Variable } & \multicolumn{2}{|l|}{ Adjusted analysis ${ }^{a}$} \\
\hline & $\begin{array}{l}\text { Without multiple imputation } \\
\text { for missing values }\end{array}$ & $\begin{array}{l}\text { With multiple imputation } \\
\text { for missing values }\end{array}$ \\
\hline & \multicolumn{2}{|l|}{$O R^{b}(95 \% C I)$} \\
\hline \multicolumn{3}{|l|}{ At-risk drinker } \\
\hline 3 months (control group, $n=309$, intervention group, $n=246$ ) & $0.41(0.22-0.75)^{* *}$ & $0.45(0.25-0.81)^{* *}$ \\
\hline 12 months (control group, $n=299$, intervention group, $n=222$ ) & $0.68(0.36-1.26)$ & $0.75(0.42-1.36)$ \\
\hline \multicolumn{3}{|l|}{ One or more heavy drinking days in past 7 days } \\
\hline 3 months (control group, $n=296$, intervention group, $n=234$ ) & $0.46(0.22-0.99)^{*}$ & $0.45(0.21-0.96)^{*}$ \\
\hline \multirow{2}{*}{12 months (control group, $n=294$, intervention group, $n=213$ ) } & $0.89(0.4-1.97)$ & $0.88(0.41-1.90)$ \\
\hline & \multicolumn{2}{|c|}{$\mathrm{RR}^{\mathrm{c}}(95 \%)$ intervention/control } \\
\hline \multicolumn{3}{|l|}{ Risk score (range 0-7) } \\
\hline 3 months & $0.77(0.63-0.94)^{* *}$ & $0.82(0.68-0.99)^{*}$ \\
\hline 12 months & $0.89(0.73-1.09)$ & $0.94(0.78-1.13)$ \\
\hline \multicolumn{3}{|l|}{ Number of drinks in past 7 days } \\
\hline 3 months & $0.79(0.70-0.90)^{* * *}$ & $0.79(0.70-0.90)^{* * *}$ \\
\hline 12 months & $0.87(0.76-0.99)^{*}$ & $0.86(0.76-0.98)^{*}$ \\
\hline
\end{tabular}


variable, study site, age and gender. ${ }^{b}$ Logistic regression mixed model with the Stata xtlogit procedure. ${ }^{c}$ Negative binomial mixed model for 'number of drinks in past 7 days' with the Stata xtnbreg procedure and Poisson mixed mode for 'risk score' with the Stata xtpoisson procedure.

Despite these limitations, the HLAYA study is one of the first to show that asking about health behaviors and providing information and feedback about alcohol use is associated with reductions in at-risk drinking and amount of drinking in an older population in primary care settings. Even simply providing information on recommended drinking limits targeted to older adults may be enough to cause large reductions in both at-risk drinking and amount of alcohol use. This study also found that older at-risk drinkers typically had multiple risks, most because of combined use of alcohol and medications and/or use of alcohol in the presence of comorbidity. Further evaluation of approaches to reduce risks associated with alcohol consumption in older adults who take multiple medications and have comorbidities is still needed, particularly in primary care settings where most of this growing population receives health care.

\section{Trial registration}

clinicaltrials.gov; Identifier: NCT00379093 http://www. clinicaltrials.gov/

\section{Declarations of interest}

None.

\section{Acknowledgements}

This study was supported by R01 AA013937, K23 AA00270, and K24 AA15957 (awarded to Dr Moore) from the National Institute of Alcohol Abuse and Alcoholism, P30AG021684 from the National Institute on Aging (Dr Moore), P30MH082760 from the National Institute of Mental Health (Dr Tang), the Medical Student Training in Aging Program, funded by the National Institute on Aging (T35 AG026736), and the John A. Hartford Foundation (Ms Gill and Ms Chen), Special Fellowship in Advanced Geriatrics, Veterans Affairs Greater Los Angeles Healthcare System (Dr Lin). Data management activities were supported by the UCLA Claude Pepper Older Americans Independence Center funded by the National Institute on Aging (P30AG028748). The sponsor provided financial support for the study only and had no role in the design and conduct of the study; the collection, management, analysis and interpretation of the study; or in the preparation, review or approval of the manuscript. We gratefully acknowledge the patients and clinicians at Desert Oasis Healthcare (DOHC), Kaiser Permanente Southern California (KPSC)-Panorama City and Woodland Hills and UCLA Healthcare, without whom this study could not have been completed. We also thank Michael Mason MD for his leadership of this project at SCKP-Woodland Hills, James Miller for his recruiting efforts at DOHC and Eric Zamora BA, who both recruited subjects and conducted countless interviews. We also would like to acknowledge the assistance provided by the UCLA Academic Technology Services Statistical Consulting Group. 


\section{References}

1. Saitz R. Unhealthy alcohol use. N Engl J Med 2005; 352: 2139-40.

2. Kirchner J. E., Zubritsky C., Cody M., Coakley E., Chen, H., Ware J. H. et al. Alcohol consumption among older adults in primary care. J Gen Intern Med 2007; 22: 92-7.

3. Weyerer S., Schaufele M., Eifflaender-Gorfer S., Kohier L., Maier W., Haller F. et al. At-risk alcohol drinking in primary care patients aged 75 years and older. Int J Geriatr Psychiatry 2009; 24: 1376-85.

4. Oslin D. W., Grantham S., Coakley E., Maxwell J., Miles K., Ware J. et al. PRISM-E: comparison of integrated care and enhanced specialty referral in managing at-risk alcohol use. Psychiatr Serv 2006; 57: 954-8.

5. National Institute on Alcohol Abuse and Alcoholism. Helping Patients Who Drink Too Much: A Clinician's Guide. NIH Publication no. 07-3769. Bethesda, MD: NIAAA; 2005.

6. Merrick E. L., Hodgkin D., Garnick D. W., Horgan C. M., Panas L., Ryan M. et al. Unhealthy drinking patterns and receipt of preventive medical services by older adults. J Gen Intern Med 2008; 23: 1741-8.

7. Breslow R. A., Faden V. B., Smothers B. Alcohol consumption by elderly Americans. J Stud Alcohol 2003; 64: 884-92.

8. Linnoila M., Erwin C. W., Cleveland W. P., Logue P. E., Gentry W. D. Effects of alcohol on psychomotor performance of men and women. J Stud Alcohol 1978; 39: 745-58.

9. Vestal R. E., McGuire E. A., Tobin J. D., Andres R., Norris A. H., Mezey E. Aging and ethanol metabolism. Clin Pharmacol Ther 1977; 21: 343-54.

10. Moore A. A., Whiteman E. J., Ward K. T. Risks of combined alcohol/medication use in older adults. Am J Geriatr Pharmacother 2007; 5: 64-74.

11. Moore A. A., Morton S. C., Beck J. C., Hays R. D., Oishi S. M., Partridge I. M. et al. A new paradigm for alcohol use in older persons. Med Care 1999; 37: 165-79.

12. Moore A. A., Giuli L., Gould R., Hu P., Zhou K., Reuben D. et al. Alcohol use, comorbidity, and mortality. J Am Geriatr Soc 2006; 54: 757-62.

13. Kaner E. F., Dickinson H. O., Beyer F., Pienaar E., Schlesinger C., Campbell F. et al. The effectiveness of brief alcohol interventions in primary care settings: a systematic review. Drug Alcohol Rev 2009; 28: 301-23.

14. Fleming M. F., Manwell L. B., Barry K. L., Adams W., Stauffacher E. A. Brief physician advice for alcohol problems in older adults: a randomized community-based trial. J Fam Pract 1999; 48: 378-84.

15. Fink A., Elliott M. N., Tsai M., Beck J. C. An evaluation of an intervention to assist primary care physicians in screening and educating older patients who use alcohol. J Am Geriatr Soc 2005; 53: 1937-43.

16. Fink A., Morton S. C., Beck J. C., Hays R. D., Spritzer K., Oishi S. et al. The alcohol-related problems survey: identifying hazardous and harmful drinking in older primary care patients. J Am Geriatr Soc 2002; 50: 1717-22.

17. Fink A., Tsai M. C., Hays R. D., Moore A. A., Morton S. C., Spritzer K. et al. Comparing the alcohol-related problems survey (ARPS) to traditional alcohol screening measures in elderly outpatients. Arch Gerontol Geriatr 2002; 34: 55-78.

18. Moore A. A., Hays R. D., Reuben D. B., Beck J. C. Using a criterion standard to validate the Alcohol-Related Problems Survey (ARPS): a screening measure to identify harmful and hazardous drinking in older persons. Aging (Milan) 2000; 12: 221-7.
19. Moore A. A., Beck J. D., Babor T. F., Hays R. D., Reuben D. B. Beyond alcoholism: identifying older, at-risk drinkers in primary care. J Stud Alcohol 2002; 63: 316-24.

20. Barnes A. J., Moore A. A., Xu H., Ang A., Tallen L., Mirkin M. et al. Prevalence and correlates of at-risk drinking among older adults: the Project SHARE study. J Gen Intern Med 2010; 25: 840-6.

21. Grimshaw J. M., Shirran L., Thomas R., Mowatt G., Fraser C., Bero L. et al. Changing provider behavior: an overview of systematic reviews of interventions. Med Care 2001; 39: 112-45.

22. Fleming M. F., Barry K. L. A three-sample test of a masked alcohol screening questionnaire. Alcohol Alcohol 1991; 26: 81-91.

23. Miller W., Rollnick S. Motivational Interviewing: Preparing People to Change Addictive Behavior. New York: Guilford Press; 1991.

24. Lin J. C., Karno M. P., Tang L., Barry K. L., Blow F. C., Davis J. W. et al. Do health educator telephone calls reduce at-risk drinking among older adults in primary care? J Gen Intern Med 2010; 25: 334-9.

25. Siu A. L., Reuben D. B., Hays R. D. Hierarchical measures of physical function in ambulatory geriatrics. J Am Geriatr Soc 1990; 38: 1113-19.

26. Sobell L. C., Sobell M. B. Timeline followback: a technique for assessing self-reported ethanol consumption. In: Allen J., Litten R. Z., editors. Measuring Alcohol Consumption: Psychosocial and Biological Methods. Totowa, NJ: Humana Press; 1992. p. 71-2.

27. Ockene J. K., Adams A., Hurley T. G., Wheeler E. V., Herbert J. R. Brief physician- and nurse practitioner-delivered counseling for high-risk drinkers: does it work? Arch Intern Med 1999; 159: 2198-205.

28. Horton N. J., Kim E., Saitz R. A cautionary note regarding count models of alcohol consumption in randomized controlled trials. BMC Med Res Methodol 2007; 7: 9.

29. Laird N. M., Ware J. H. Random-effects models for longitudinal data. Biometrics 1982; 38: 963-74.

30. Schafer J. L. Analysis of Incomplete Multivariate Data. New York: Chapman \& Hall; 1997.

31. Tang L., Song J., Belin T. R., Unutzer J. A comparison of imputation methods in a longitudinal randomized clinical trial. Stat Med 2005; 24: 2111-28.

32. Fleming M. F., Barry K. L., Manwell L. B., Johnson K., London R. Brief physician advice for problem alcohol drinkers. A randomized controlled trial in communitybased primary care practices. JAMA 1997; 277: 103945.

33. Clifford P. R., Maisto S. A. Subject reactivity effects and alcohol treatment outcome research. J Stud Alcohol 2000; 61: 787-93.

34. Kypri K., Langley J. D., Saunders J. B., Cashell-Smith M. L. Assessment may conceal therapeutic benefit: findings from a randomized controlled trial for hazardous drinking. Addiction 2007; 102: 62-70.

35. Grant B. F. Prevalence and correlates of alcohol use and DSM-IV alcohol dependence in the United States: results of the National Longitudinal Alcohol Epidemiologic Survey. J Stud Alcohol 1997; 58: 464-73.

36. Falk D. E., Yi H. Y., Hiller-Sturmhofel S. An epidemiologic analysis of co-occurring alcohol and tobacco use and disorders: findings from the National Epidemiologic Survey on Alcohol and Related Conditions. Alcohol Res Health 2006; 29: $162-71$. 